\title{
Modelling of autoresonant control of a parametrically excited screen machine
}

\author{
S. Abolfazl Zahedi ${ }^{1}$, Vladimir Babitsky ${ }^{2}$ \\ ${ }^{1}$ School of Mechanical, Aerospace and Civil Engineering, University of Manchester, \\ Manchester, M13 9PL, UK, Corresponding author: Abolfazl.Zahedi@Manchester.ac.uk \\ ${ }^{2}$ Wolfson School of Mechanical and Manufacturing Engineering, Loughborough University, \\ LE11 3TU, UK, V.I.Babitsky@lboro.ac.uk
}

\begin{abstract}
Modelling of nonlinear dynamic response of a screen machine described by the nonlinear coupled differential equations and excited by the system of autoresonant control is presented. The displacement signal of the screen is fed to the screen excitation directly by means of positive feedback. Negative feedback is used to fix the level of screen amplitude response within the expected range. The screen is anticipated to vibrate with a parametric resonance and the excitation, stabilisation and control response of the system are studied in the stable mode. Autoresonant control is thoroughly investigated and output tracking is reported. The control developed provides the possibility of self-tuning and self-adaptation mechanisms that allow the screen machine to maintain a parametric resonant mode of oscillation under a wide range of uncertainty of mass and viscosity.
\end{abstract}

Keyword: parametric resonance, screen oscillation, autoresonant control

\section{Introduction}

Vibratory machines and structures are used in many industrial applications, either as components or individual pieces of equipment [1-3]. The vibrating screen is an example of a vibratory machine used to separate bulk materials from a mixture of particles of different sizes. These machines can perform simple safety screening as well as accurately grade powders or granules. For example, sand, gravel, river rock, crushed rock and other aggregates are often separated by size using vibrating screens. To increase the intensity and energy efficiency, resonant modes will be effective for vibratory screen machines. In addition to giving a high-efficiency performance, operating a 
system in a resonant mode also provides the additional advantages of being able to separate large particles from fine, and dry from wet. However, the practical use of resonant modes is hindered by difficulty of the resonant tuning under the variable processing loads.

In general, two types of resonant response can be distinguished in mechanical systems, forced resonance and parametric resonance (PR). If the frequency of an external periodic excitation is close to the natural frequency of a system, the system will experience forced resonance. Parametric oscillations occur in systems having time-varying (periodic) parameters. In PR the amplitude of the response grows exponentially, and linear damping elements do not help to saturate this growth. In contrast, forced resonance is caused by an additive driving force. Compared with the forced resonance response, PR is characterized by a much higher intensity within a broad range of frequencies. This type of oscillation occurs in a wide variety of engineering applications and when the excitation term appears as the time dependence parameter in the governing equations of motion [4].

The PR condition can display various behaviours including periodic, quasi-periodic, non-periodic and chaotic behaviours [5]. Moreover, resonance often occurs along with saturation phenomena. In particular, when the secondary system enters into PR it functions as an energy absorber by draining energy from the external excitation through the primary system. This entails a large increase in the amplitude of the displacements of the secondary system, whereas the oscillation amplitude of the primary system is maintained as almost constant.

The idea of applying PR to drive screen motion was patented in 2009 [6]. The new generation of machines using PR have some advantages over conventional machines, in which the transverse oscillations are excited directly. PR-based machines have been demonstrated to have large amplitudes of high-frequency oscillations with a high rate of energy transfer, wide resonantfrequency region, low sensitivity to damping, in-plane coupling properties, lateral oscillations and convenient vibration insulation. This type of oscillation has been used in screen machines for the transportation and separation of granular media, which are characterized by larger amplitude values and insensitivity to the dissipation level across a rather wide range of viscosities [7].

PR demonstrates large amplitudes of high-frequency lateral oscillations, robust vibration operation, and the ability to process naturally wet, fine granular material. Although implementation of the PR regime is generally the most efficient method to drive the screen 
machine, its maintenance is subject to difficulties, especially in systems with high Q-factors and varying parameters and loads [8]. Dangerous high-amplitude oscillations may arise in the system, potentially leading to catastrophic failures (Figure 1). This is due to the strong sensitivity of resonant tuning to parameters and structural deviations. The problem of managing a finely tuned resonance control is drastically complicated when nonlinear factors, unpredictable variable loads, or limited excitation forces produced by the energy source occur.

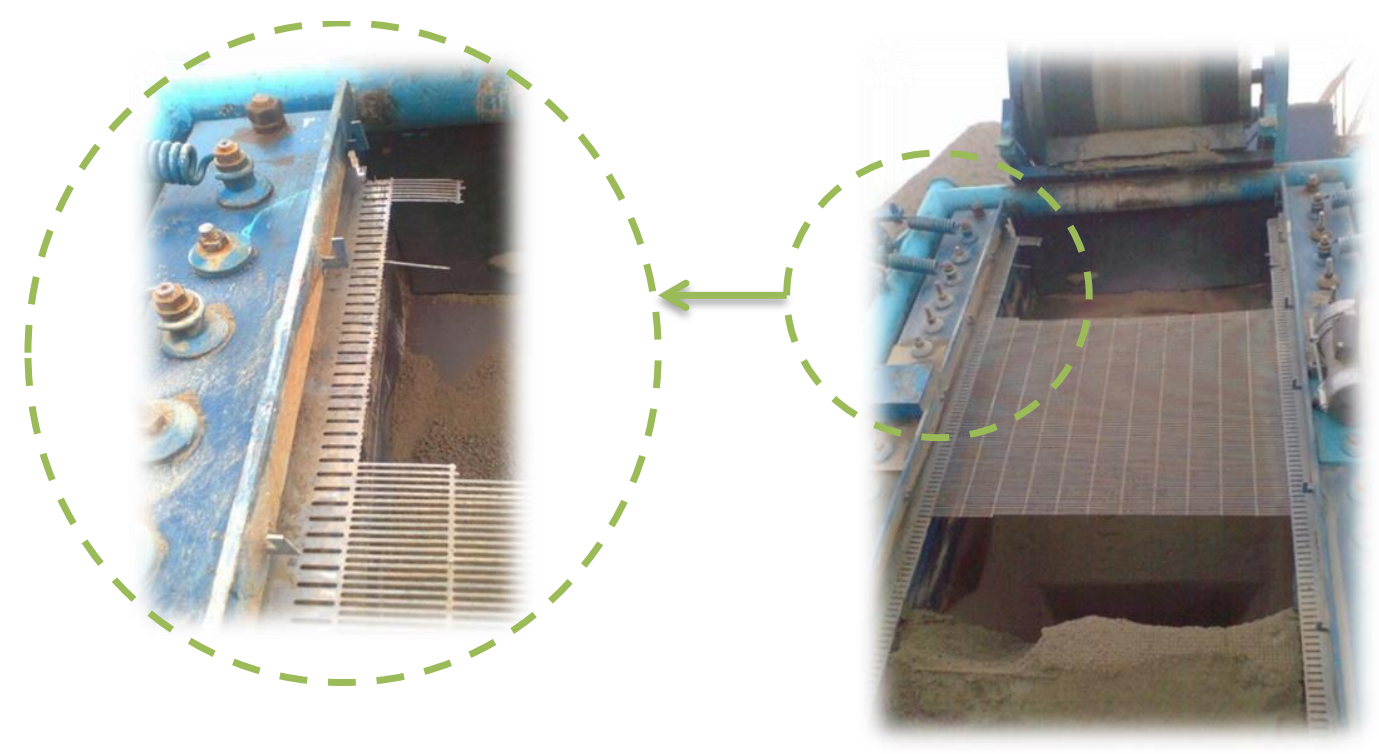

Figure 1: Screen failure due to high-amplitude oscillations

In most resonance-related research, the motivation is to develop techniques to avoid or minimize the damaging effects of PR on the structure operating within the expected range of excitation frequencies and forces [9-10]. Few potential methods have been tried in the past to make the amplitude elevate to the desired level, and make the PR oscillations well-ordered, to create a stable regime. Okuma et al. [11] and Yabuno et al. [12] researched the nonlinear characteristics of PR induced in a cantilever beam by a linear feedback control, based on the passive pendulum control method. The study by Oueini and Nayfeh, [13] showed that PR-induced high amplitude vibrations cannot be fully prevented by linear or quadratic feedback control. Chen et al. [14] implemented combined linear and nonlinear-velocity feedback control to suppress the principal PR in a flexible cantilever beam structure. These studies were mainly dedicated to the use of lumped-parameter vibration absorbers to suppress the principal PR in structures, however, implementation of such absorbers is subject to limitations for large flexible structures like vibrating screens. It is 
noteworthy, that due to geometrical and non-linear oscillations in the screen drive motion, the system follows several possible regimes of vibration when the same frequency control is applied. Therefore, the actual steady-state response of the system under frequency control is determined by the history of the control and limited by the use of frequency control to achieve steady performance of the screen system.

The central problem in PR applications is to control the oscillation instability with the aim of keeping the amplitude at a desired level, and making the PR oscillations well-ordered to create a stable response. The autoresonance method is used in the current research for screen systems to control the system response due to its ability to deal with nonlinearity and its robustness when confronting systems with a wide range of uncertainties. This method is well established for machining applications [1, 15-20].

The paper is organized as follows: in Section 2, the dynamic model of the screen is described in detail; the principle of autoresonant control is introduced in Section 3; and in Section 4, features of the tuning system are explained, followed by two case studies of mass and viscosity uncertainties.

\section{Dynamic model of the screen}

The PR-based vibrating screen machine manufactured by Loginov and Partner Mining Company (Kiev, Ukraine) is presented in Figure 2(a). The machine includes: the vibrator (1), the base (2), the beams fastening the sieve (3), the side springs (4) and the sieve plate (5) (which is mainly under the lid). The schematic diagram of the dynamic model of the machine is shown in Figure 2(b). This mechanism is simplified by synchronously moving end mass in opposite horizontal directions, while the string is able to oscillate laterally. These two modes of oscillation are coupled because the tensile force depends on both the longitudinal displacements of the masses and the transverse displacements of the string (the latter dependence is nonlinear).

The dynamic model of the PR-based vibrating screen (Fig. 2(b)) represents an initially stretched system of two equal masses connected by an elastic string. The end masses, which can move horizontally, are also connected with a rigid frame by the side springs. The longitudinal oscillations are excited by harmonic external forces acting synchronically on the left and right 
masses in opposite horizontal directions. Under certain conditions this motion results in transverse oscillations of the string. For simplicity, planar motion of the screen is assumed and the treated granular material action is reflected by linear viscosity.

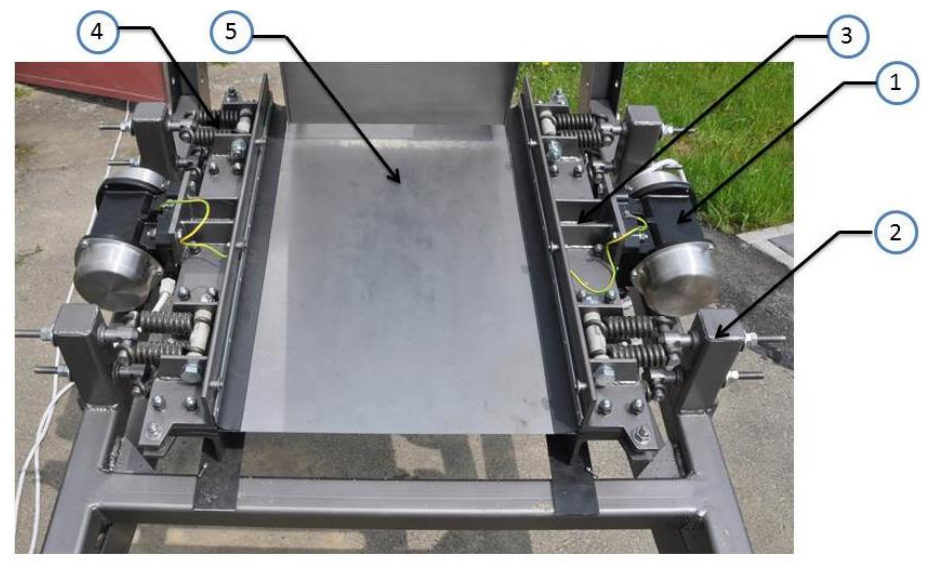

(a)

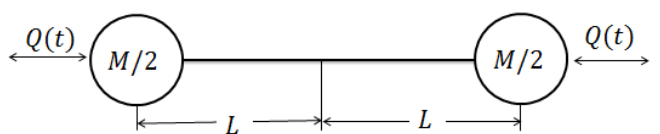

(b)

Figure 2: The vibrating screen machine (a) vibrator (1), base (2), beams fastening the sieve (3), side springs (4) and the sieve plate (5) and its dynamic model (b), after [7]

The dynamic model of two ordinary differential equations coupled by the geometric nonlinearity as presented by L. Slepyan and V. Slepyan [7] is considered the equation of motion in this research. Eqs. 1 are subject to longitudinal and transverse oscillations.

$$
\left\{\begin{array}{l}
M \frac{d^{2} u(t)}{d t^{2}}+\beta \frac{d u(t)}{d t}+T_{1}(t)=Q \cos (\gamma t+\varphi) \\
\rho \frac{d^{2} v(x, t)}{d t^{2}}+\alpha \frac{d v(x, t)}{d t}-T(t) \frac{d^{2} v(x, t)}{d x^{2}}=0
\end{array}\right.
$$

In these equations, $M$ and $\rho$ are the end mass value and mass per unit length, respectively; $\beta$ and $\alpha$ are the viscosity numbers associated with the longitudinal and transverse oscillations; $u(t)$ is the displacement of the right end mass $(-u(t)$ corresponds to the left), $v(x, t)$ is the transverse displacement of the string at the first mode of vibration. The equation $v(0, t)=\omega(t)$ shows that the transverse displacement depends only on time. The excitation signal $(Q \cos (\gamma t+\varphi))$ is noted with $Q$, as an excitation amplitude, $\gamma$ as a frequency and $\varphi$ as a phase. The dynamic behaviour of the treated material depends on weight and effective viscosity. In particular, the maximal weight of the material placed on the sieve plate determines the $\rho$ parameter. The current model is 
described by a system of nonlinear differential equations, tracing the influence of the screen structure, setting, granular material weight and effective viscosity.

The relation between tensile force $T_{1}(t)$, and total force $T(t)$ in the string was expressed by the following equations [7]

$$
\begin{aligned}
& T_{1}(t)=k\left(u(t)+\frac{\pi^{2}}{16 L} \omega^{2}(t)\right) \\
& T(t)=T_{0}+T_{1}(t)
\end{aligned}
$$

where $T_{0}$ is initial tensile force, $L$ is the string length and $k$ is the side spring stiffness. Replacing Eqs. 2 in Eqs. 1 and rearranging them, results in:

$$
\left\{\begin{array}{l}
M \frac{d^{2} u(t)}{d t^{2}}+\beta \frac{d u(t)}{d t}+k u(t)=Q \cos (\gamma t+\varphi)-k \frac{\pi^{2}}{16 L} \omega^{2}(t) \\
\rho \frac{d^{2} \omega(t)}{d t^{2}}+\alpha \frac{d \omega(t)}{d t}+\frac{\pi^{2}}{4 L^{2}}\left(T_{0}+k u(t)\right) \omega(t)=\frac{\pi^{2} k}{64 L^{2}} \omega^{3}(t),
\end{array}\right.
$$

where the primary system is externally excited by a harmonic force, and the secondary system is parametrically excited. The parametric excitation term $\left(\frac{\pi^{2}}{4 L^{2}}\left(T_{0}+k u(t)\right)\right)$ results in the observed PR response in the screen motion. This system represents a system of Mathieu-Hill equations, where the effects of damping are added [21].

During the resonant mode of operation, the displacement of the sieve plate increases, and the screen oscillates with higher amplitude. PR has the ability to exhibit higher mechanical amplification than direct resonance when operating in favourable conditions. The displacement responses of the system are shown in Figure 3, while the frequency of external excitation is close to twice that of the natural frequency of the string equation. The steady state responses of the system show that small oscillations of the mass $(u(t))$ lead to a large amplitude of the sieve plate $(\omega(t))$ when PR is fulfilled in the screen machine. The ratio of maximum longitudinal displacement to transverse displacement level $\left(\frac{u(t)}{\omega(t)}\right)$ is around 10, showing dramatic improvement of the screen efficiency. Implementation of PR allows the company to reduce costs and equipment downtime, and enables them to gain a market advantage by being able to make fine separations, and provide higher purity and more precise sizing control of the product than a traditional vibrating 
screen. With a traditional vibration screen, the dissipation is highest at the centre, and the material gathers there, but with PR not only is the amplitude larger for a given amount of input energy, but the dissipation is better distributed, so material does not clump. Both initial conditions and constant parameter values, used to produce this figure, are noted as $M=400 \mathrm{~kg}, k=400 \mathrm{Nm}^{-1}$, $Q=0.7 \mathrm{~N}, \gamma=1 \mathrm{~s}^{-1}, \alpha=4 / 3 \mathrm{~N} . \mathrm{s} \mathrm{m}^{-1}, \beta=8 / 3 \mathrm{~N} . \mathrm{s} \mathrm{m}^{-1}, T_{0}=1 \mathrm{~N}, u(0)=0.0025 \mathrm{~m}, \omega(0)=-0.06$ $\mathrm{m}$ and $\dot{u}(0)=\dot{\omega}(0)=0$.

(a)

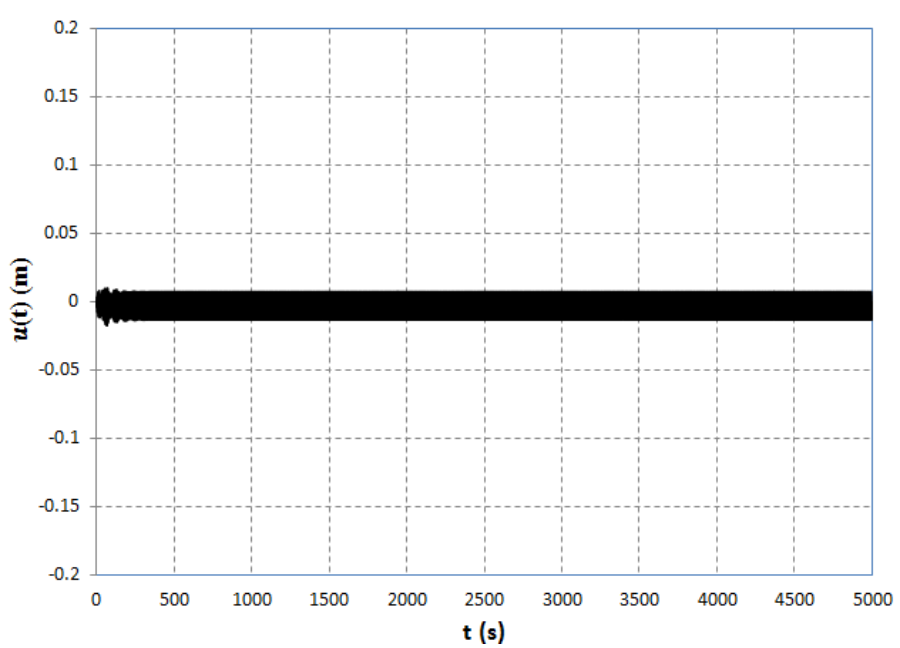

(b)

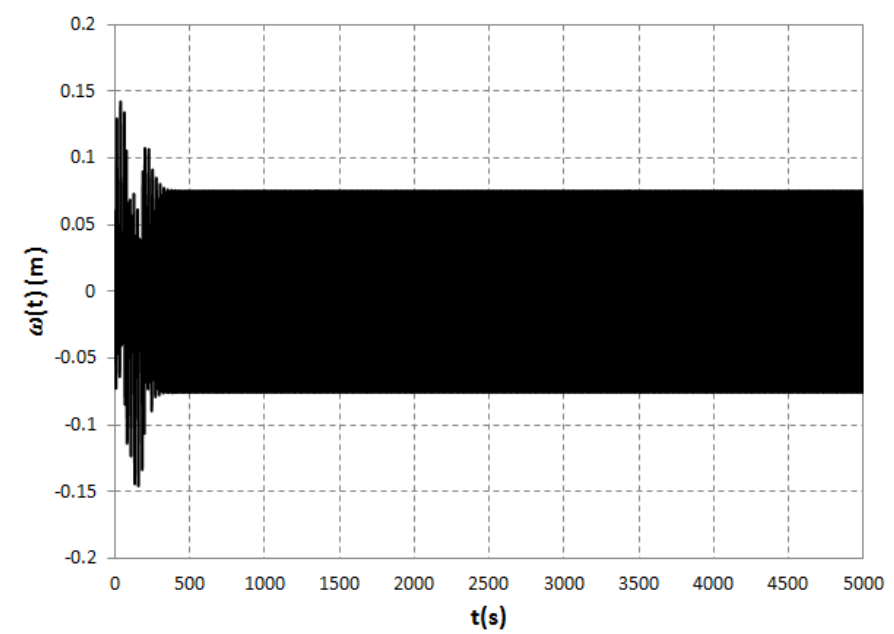

Figure 3: The longitudinal $u(t)$ (a) and transverse $\omega(t)$ (b) displacements of screen machine variables

The amplitude-frequency characteristics for the parametrically excited non-linear system with two degrees of freedom are shown in Figure 4. For the particular system chosen, the harmonic PR is predicted to occur at a frequency around $1 \mathrm{~s}^{-1}$. The natural frequency of the sieve plate is around $0.5 \mathrm{~s}^{-1}$ while the external excitation frequency is around $1 \mathrm{~s}^{-1}$. Therefore, while the first system 
vibrates in normal resonance mode the second operates at twice its natural frequency. The fundamental resonance peaks for longitudinal $u(t)$ and transverse $\omega(t)$ displacements were observed to occur at the same frequency. It can clearly be seen that the peak value in the amplitudefrequency curve is very sharp which makes fixed frequency control insufficient.

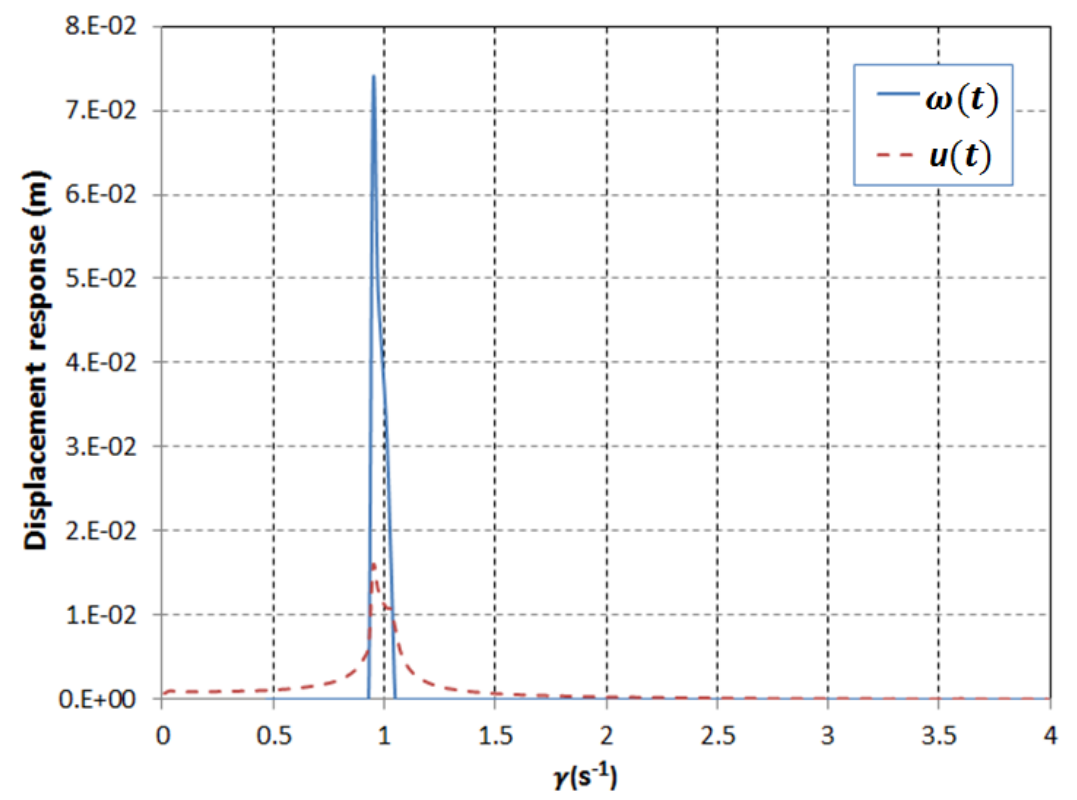

Figure 4: Illustration of amplitude-frequency characteristics of the nonlinear screen system

The loss of stability (quasi-periodic or chaotic) of a vibrating sieve plate can occur at detuning values corresponding to changing screen parameters such as mass or viscosity, or excitation parameters such as phase or frequency. In the next section the principle of the autoresonant control method is presented, which is used for the stabilization of PR.

\section{The principle of autoresonant control}

The main purpose of a control system is to keep the vibrations at a specified level during the oscillation process. In autoresonant control the resonant domain of oscillation is automatically maintained by positive feedback using transformation (phase shift) and an amplification signal from a movement sensor of the sieve. This is based on the fact that during resonance, the phase lag between the vibration of the working element (screen) and the applied excitation force is constant. This control method does not have a prescribed excitation frequency. A general schematic of a control feedback system is presented in Figure 5. 


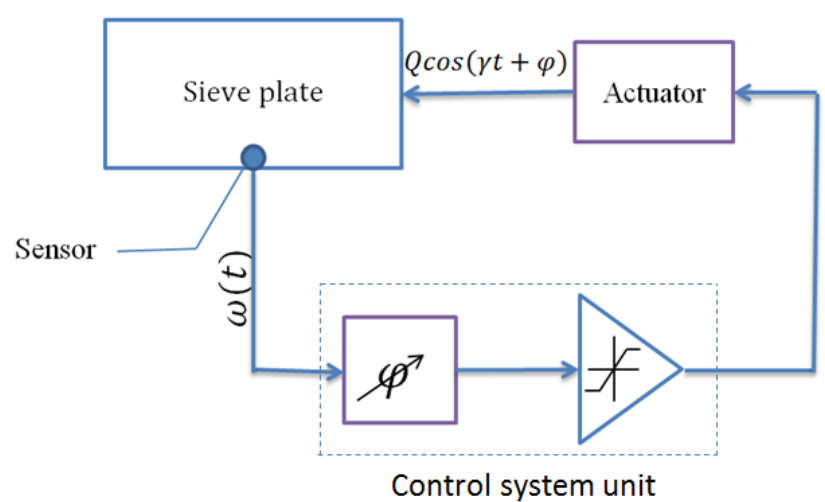

Figure 5: General schematic of control feedback system

The control feedback system includes a sensor, control system unit and actuators. The input of the system is a sensor signal measuring the sieve vibration $\omega(t)$, and the output is $Q \cos (\gamma t+\varphi)$, the excitation force generated by machine actuators. In the case of the described model the sensor is attached to the screen machine to measure the mechanical characteristics of the oscillations (displacement of the sieve plate). The signal is used by the control system unit, and generates a control signal, in its simplest form, shifting the phase of the vibration signal from the sensor and amplifying its magnitude. The powerful signal produced feeds a synchronous type power actuator which transforms the signal to the excitation force $(Q \cos (\gamma t+\varphi))$ with the same frequency and phase shift calculated by a control system unit. This force oscillates the beams that fasten the sieve plate.

The schematic diagram of autoresonant control is shown in more detail in Figure 6, and demonstrates positive feedback including a phase shifter, limiter and filter. The parameters of selfsustaining vibration can be controlled by changing the phase shift and/or the limitation level in the feedback circuit. 


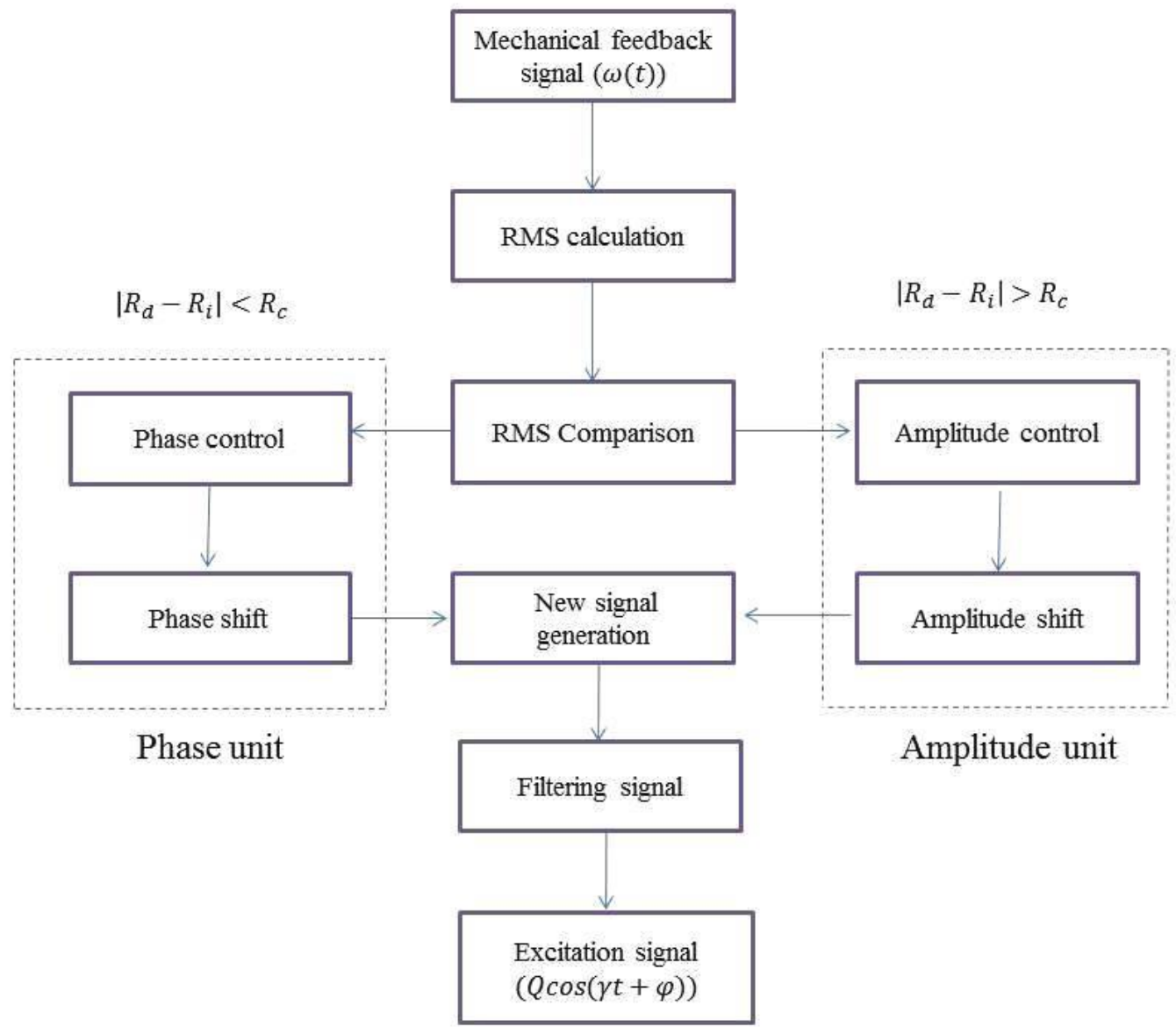

Figure 6: Schematic diagram of the autoresonant control, $R_{d}$ is the desired RMS, $R_{c}$ is the critical RMS, and $R_{i}$ is the present RMS control signal

In order to compute the root mean square (RMS) value, the following equation is used:

$$
R=\sqrt{\frac{1}{T} \int_{0}^{T} \omega(t)^{2} d t}
$$

where $R$ is the RMS of a control cycle, $T$ represents the period of a control cycle which equals 100 oscillation periods in this case, and $\omega(\mathrm{t})$ denotes sieve vibration. The $R$ value remains constant within one control cycle and will not change until the beginning of the next one. In Figure $6, R_{d}$ represents the desired RMS of a sensor signal (fixed at 0.08 in this case); $R_{c}$ is the critical RMS value fixed at $5 \% R_{d}$ and $R_{i}$ is the RMS of the current control signal.

In the condition $\left|0.08-R_{i}\right|<0.04$ the phase unit is executed. This unit includes phase control and phase shift blocks designed to shift the system into the PR domain of oscillation according to the change in the RMS of a control cycle. In this unit, the RMS of each control cycle is calculated and compared with the RMS of the previous cycle $\left(R_{i-1}\right)$. When $R_{i}>R_{i-1}$, the phase will be 
increased by $0.5^{\circ}$; otherwise $0.5^{\circ}$ will be subtracted from the phase. There is a domain for changing the phase, discussed later. Therefore, the phase control signal is constantly changed by $0.5^{\circ}$ between the threshold values. The inputs to the phase control block are the RMS values and a parameter called counter. Counter values are recorded as the number of oscillation periods that form one control cycle. The output from this block is the actual phase shift value $(\varphi)$ determined by the phase control algorithm which is further processed by the phase shifter block.

The phase shifter block is designed to add the phase $(\varphi)$ calculated from the phase control block to the control signal. Since this block is only applicable to a square wave signal, a sinusoidal wave is transformed to a square wave with a unity gain. Basically, for a symmetric sinusoidal signal centred around zero, this block converts the positive half wave into a positive straight unity line, and transforms the negative half wave into a negative straight unity line. Therefore, a square wave is generated with exactly the same frequency as the original sensor signal. The counter values determine each oscillation period. The detail of converting a sinusoidal wave to a square wave is well presented in $\mathrm{Li}$ [22] and Voronina [23] $\mathrm{PhD}$ theses. The newly generated square wave can be further processed by an amplifier.

An amplitude unit is designed to control the PR domain automatically, by adjusting the amplification gain in order to provide extra power to the actuator to maintain the vibration at the desired level during operation. This unit operates in the condition $\left|0.08-R_{i}\right|>0.04$ and consists of two blocks, amplitude control and amplitude shift. There are two inputs to this unit which are identical to those of the phase unit, RMS and counter value. The amplitude control algorithm provides automatic control of the limiter threshold to keep the RMS value of the reference signal at the same level during oscillation. It is performed by a nonlinear transducer with a variable level of saturation. The new amplitude $Q_{\mathrm{i}+1}$, was calculated based on following equation:

$$
Q_{i+1}=Q_{i} \frac{R_{d}}{R_{i+1}}
$$

where $Q_{\mathrm{i}}$ is the amplitude of the present control cycle signal, $Q_{i+1}$ is the elaborated control signal value, and $R_{i+1}$ is the RMS of a sensor signal obtained from the next control cycle. If $R_{i+1}$ is greater than $R_{d}$, then the amplitude $Q_{i+1}$ will decline and vice versa. The value $Q_{i+1}$ would be set if, and only if, $R_{i+1}$ differs from $R_{d}$ by more than $5 \%$, otherwise the $Q_{i}$ value would be retained. The output from this block is the calculated amplitude value $(Q)$ which is fed into the amplifier shift block. The amplitude shift block, as with the phase shift block, needs a square wave, and is 
designed to amplify the shifted square wave by multiplying by a gain $(Q)$ calculated from the amplitude control block.

In order to rectify (smooth) the amplified square wave and generate a quasi-sine wave, which eliminates the high-frequency components, a second-order low-pass filter is used [18-20]. The transfer function of the second-order low-pass filter used in this case is as follows:

$$
G_{s}=\frac{1}{s+2000}
$$

where $s$ is the complex variable. This passes low-frequency signals but attenuates signals with frequencies higher than the cut-off frequency. The excitation signal is applied to a synchronous type power actuator to produce an excitation force with the same frequency and phase shift calculated for the vibrating system.

To sum up for every control cycle, the level of change in oscillation is evaluated as a RMS value and then the phase is shifted by the phase control unit and the amplitude by an amplitude control block. Basically, the amplitude control is applied when the RMS of a sensor's signal is detected to deviate of more than 5\% from the desired RMS; otherwise, phase control is used by the system to shift it to a PR oscillation domain. Therefore, typically, both amplitude and phase control signals change during the operation and keep the vibration level of the screen oscillation at the desired value. As a result, realisation of autoresonant control is achieved with positive feedback, containing phase shifter, limiter and filters. The total transformation of a signal in the controller can be described in operator form as:

$$
f(t)=F[\omega(t) \exp (j \varphi)]
$$

where nonlinear characteristics $F$ include the limitation and filtering of the RMS signal, $\varphi$ is the phase shift due to action of the phase shifter, and $j^{2}=-1$. After harmonic linearization of Eq. (7) a complex amplitude of vibration is achieved as follows:

$$
\tilde{u}=Z\left(a_{1}\right) \tilde{a}_{1} \exp (j \varphi)
$$

where $Z\left(a_{1}\right)$ is a coefficient of harmonic linearization for the limiter. A maximum response of the limiter is fixed to $Q$, i.e.

$$
|F[\omega(t) \exp (j \varphi)]| \leq Q
$$


The periodic function $f(t)$ in this case, cannot have a fundamental amplitude exceeding $4 Q / \pi$ [19]. The frequency and amplitude of the vibration are determined by the parameters of the mechanical system and feedback. Self-adaptation and self-tuning are the principle features of an autoresonant control system. This system automatically changes its regime of function in order to have the maximum dynamic effect. It always follows the state that brings the system to resonance independence in which the parameters are changed.

It is known that the nonlinear term influences the resonant frequency of the system. Eqs. 3 have stable solutions in a domain of the problem parameters due to the presence of the nonlinearity in the background. In this case, coupling plays the role of the automatic stabiliser. It is remarkable that in the case of resonant excitation, where the external force frequency is twice the frequency of the free longitudinal oscillations, the amplitudes are independent of the viscosity and the nonlinearity bounds the amplitudes.

In order to excite the autoresonant control, the possibility of phase and amplitude shift needs to be found and set. The range of change in amplitude and phase is calculated and used as the boundary of the phase and amplitude control units. In the next section the amplitude and phase limits to keep the PR mode of oscillations stable are discussed. Setting these values allows the desired level of oscillation to be approached.

\section{Setting the boundaries of the phase and amplitude}

The first step in the autoresonant control system is tuning the system for the PR domain. This is done by accomplishing the initial preparation procedure. The procedure allows identification of the optimal phase shift in the system (the phase shift gives a stable PR response). To define the optimal phase shift, the amplitude-phase characteristic of the closed loop system has to be obtained for control signals. This is achieved by slowly changing the phase shift to provide the full range from $0^{\circ}$ to $360^{\circ}$ in the system and tracing the screen oscillation. The increment step of $0.5^{\circ}$ is fixed for phase shift in each numerical model, and the amplitude peak value after initial oscillations was extracted. Figure 7 represents the results of autoresonant excitation in the closed loop control system with positive feedback under various phase shifts (provided by the phase shifter), but with the same level of limiter threshold. 


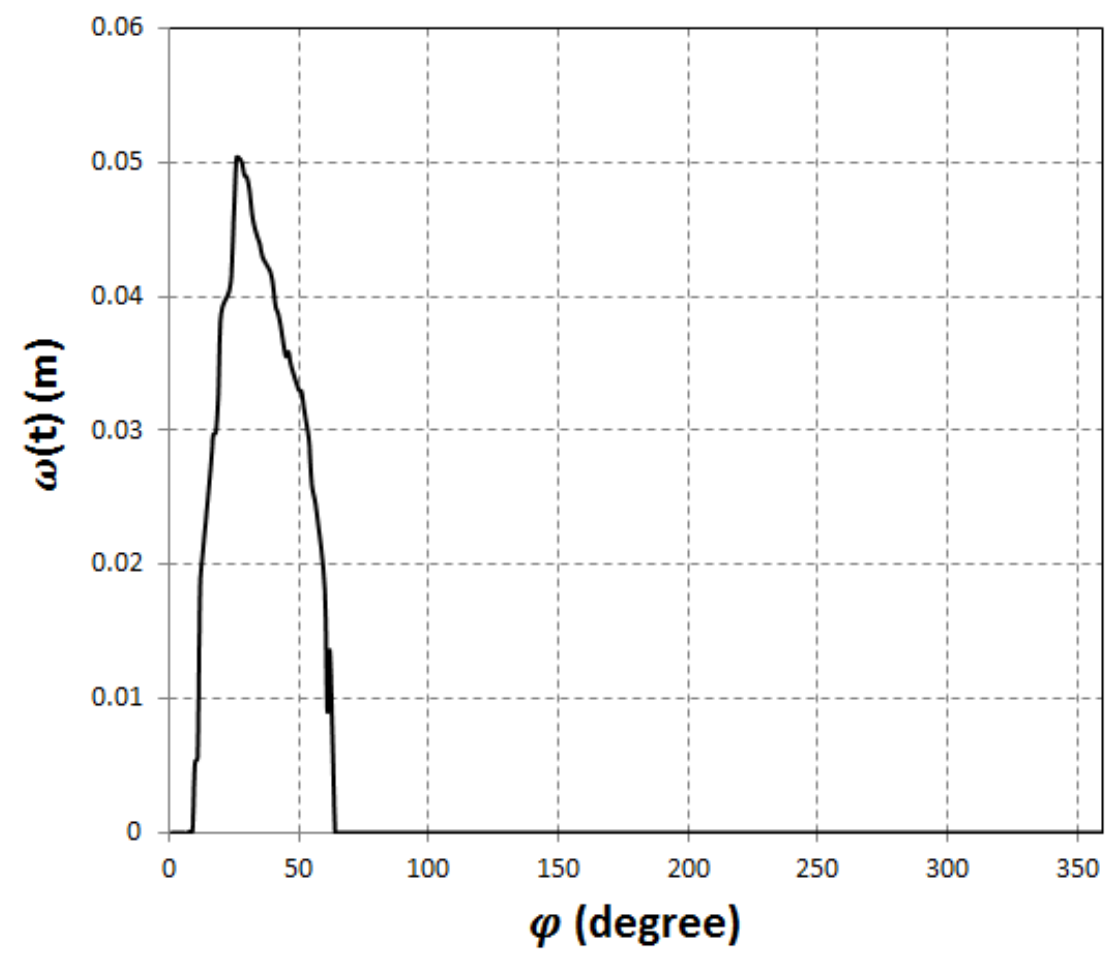

Figure 7: Amplitude-phase characteristic of the loaded system

The amplitude-phase characteristic gently slopes near the resonance and is smoother than the amplitude-frequency curve presented in Figure 4, which provides easier access to the control design. As shown in Figure 7, the phase domain between $7^{\circ}$ and $65^{\circ}$ in the feedback loop allows the tuning control system to be in the stable PR state. By setting the phase angle at $27^{\circ}$ the maximum screen amplitude is reached. The response of the screen stopped the oscillation at phases smaller than $7^{\circ}$ or larger than $65^{\circ}$. The whole control process can be realized within a domain and to maintain it, any bifurcation has to be avoided. In the test the following parameters were used:

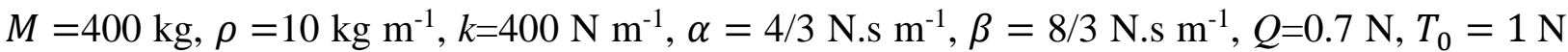
with the initial conditions of $u(0)=0.0025 \mathrm{~m}, \omega(0)=-0.06 \mathrm{~m}$ and $\dot{u}(0)=\dot{\omega}(0)=0$.

To identify the threshold of the amplitude control unit the phase shifter control signal is kept between $7^{\circ}$ and $65^{\circ}$ and the threshold of the amplitude limiter is varied from $0 \mathrm{~N}$ to $2 \mathrm{~N}$. Altering the limiter threshold permits control of the vibration level. The increment step of $0.01 \mathrm{~N}$ was kept for $Q$ (amplitude excitation).

Figure 8 presents the amplitude of the screen system over the level of excitation change. The purpose of this test was to determine the interval of the control characteristic, where the control 
signal causes linear dependence of the reference RMS signal. The minimum level of $Q$ for excitation of the screen is around $0.48 \mathrm{~N}$ while setting the values of the threshold to larger than $1.22 \mathrm{~N}$ lead to a chaotic response. Therefore, $0.48 \mathrm{~N}<Q<1.22 \mathrm{~N}$ is the limit of the output control signal amplitude. Each time $Q$ is increased in this domain the level of screen displacement is increased, however, where $\mathrm{Q}>1.22 \mathrm{~N}$ the level of vibration cannot be controlled properly.

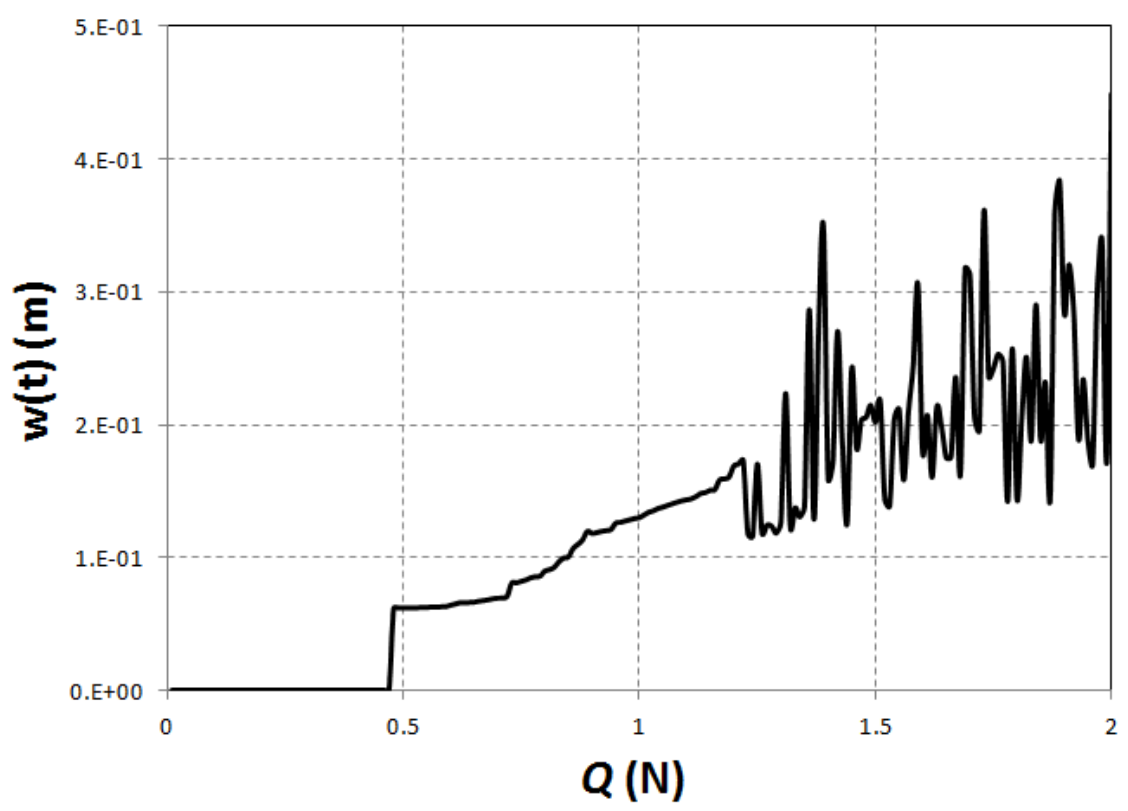

Figure 8: Threshold of the amplitude limiter

The control unit is now defined to limit the phase and amplitude response of the system in a proper domain. The performance of the control evaluation for two case studies is presented in the next section.

\section{The performance of the control system}

In Eqs.3, $\rho$ and $\alpha$ denote the mass per unit length and the viscosity, respectively, associated with transverse oscillations. When the mass or viscosity change, the resonance frequency of the system changes. Thus, to obtain the PR regime which is the most efficient domain in which to drive the screen machine, the excitation signal should be changed. The results of autoresonant control, with positive feedback, using screen displacement as a mechanical control signal are studied under mass and viscosity variation with the same level of limiting and phase. In particular, the induction of $\mathrm{PR}$ is achieved by shifting the system into the principal PR region, that is, where the frequency of the parametric excitation is approximately equal to twice the natural frequency of the string 
system. It is notable that the values of mass and viscosity should be considered in the acceptable range to have oscillation in the system.

\subsection{Case study 1: Changing the screen mass}

Figure 9 shows the ability of the displacement control to control the level of vibration under conditions of mass uncertainty. For illustrative purposes, the mass values are monitored together with the displacement of the screen. The level of changing mass ( $\rho$ in Eqs.3) varies from $6 \mathrm{~kg}$ to $10 \mathrm{~kg}$ (Fig. 9(a)). The other parameters in the system are fixed: $M=400 \mathrm{~kg}, k=400 \mathrm{Nm}^{-1}, \alpha=4 / 3$ N.s $\mathrm{m}^{-1}, \beta=8 / 3 \mathrm{~N} . \mathrm{s} \mathrm{m} \mathrm{m}^{-1}, Q=0.7 \mathrm{~N}, T_{0}=1 \mathrm{~N}$ with the initial conditions of $u(0)=-0.0025 \mathrm{~m}$, $\omega(0)=0.06 \mathrm{~m}$ and $\dot{u}(0)=\dot{\omega}(0)=0$. A time of $10^{5}$ s elapses between each mass increment. Figure 9(b) describes the response of the screen system over the selected time. The control system uses the RMS value of the displacement signal as a control signal, which is shown in Fig. 9(c) as a solid line; the dashed line depicts the desired value of the RMS of the displacement signal. The desired RMS value was defined as $0.08 \mathrm{~m}$, corresponding to the proper amplitude of oscillation of the loaded system. The derivation of the RMS from the desired value becomes nearly constant by changing the mass from $6 \mathrm{~kg}$ to $10 \mathrm{~kg}$. The phase and amplitude shifter signals are presented in Fig. 9(d) and Fig. 9(e). The phase shift signal is in radians, therefore, 0.485 phase shift is equal to $27.80^{\circ}$. To keep the vibration at the desired level of oscillation, both amplitude and phase control signals are changed during the operation. It can be seen that when the amplitude control is applied and the amplitude value is changing, the phase shift value remains unchanged. As soon as the RMS value enters the phase control zone the amplitude control stops working and the phase control starts. By this action the control system keeps the RMS value relatively stable in spite of a considerable increase in loadings Implementation of phase control only, is insufficient to keep the desired level of vibration under such loading, and amplitude control should be considered. 
(a)
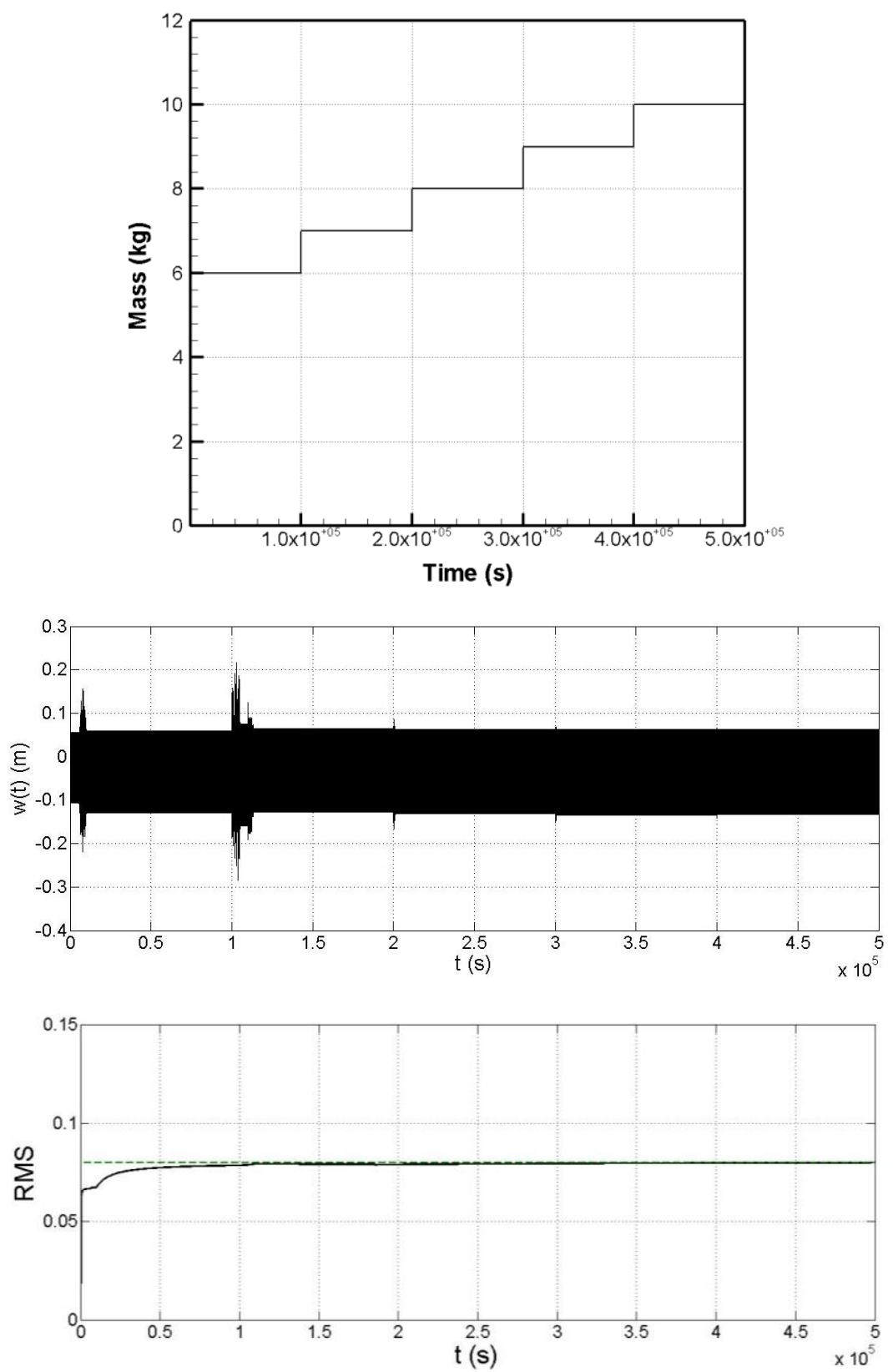

(d)

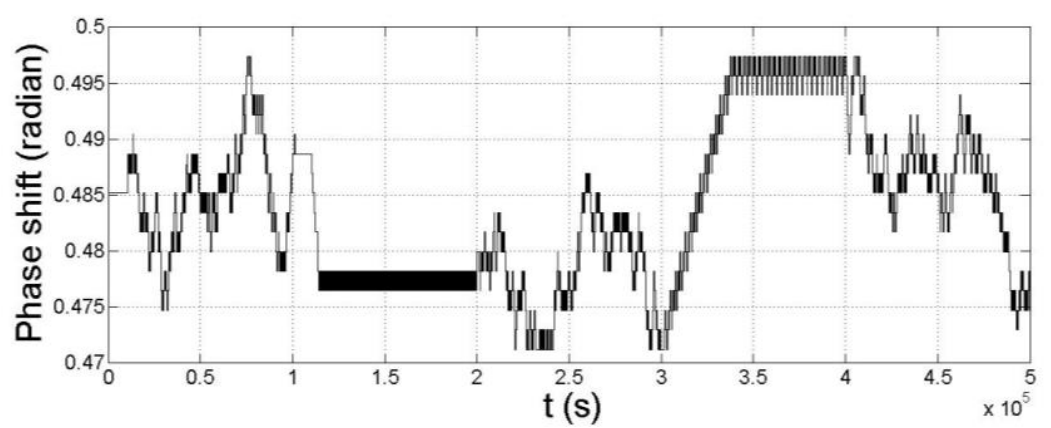


(e)

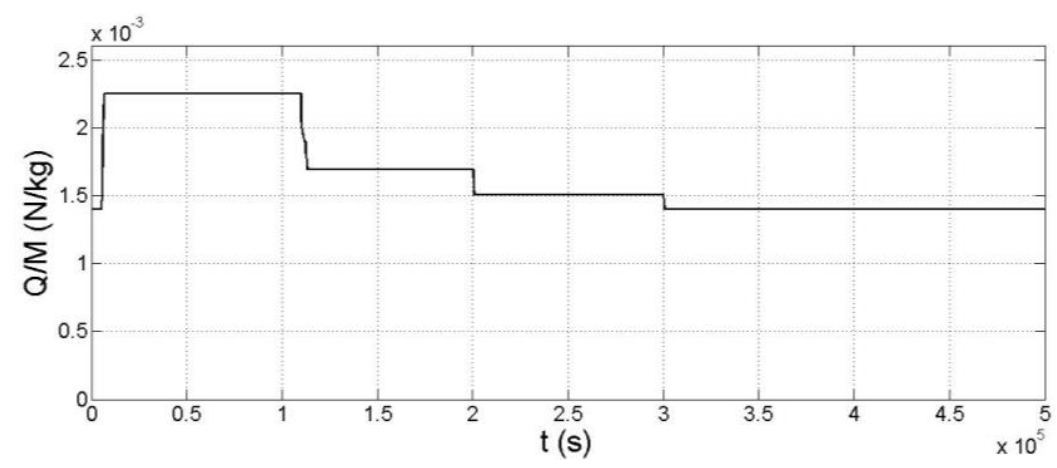

Figure 9: Performance of the autoresonant control system, mass changing (a), displacement of the screen (b), RMS value (c), phase shift signal (d) and amplitude shift signal (e)

The combined amplitude-phase control algorithm allows for the possibility of simultaneous control of the phase (phase control) and the level of oscillation (amplitude control). When the mass changes, the resonance frequency of the system changes. This results in the system being excited at different frequencies. Fixing the amplitude and phase boundary to achieve a stable PR state in the system is ensured by stabilising the oscillations of the screen system.

\subsection{Case study 2: Changing the viscosity}

The main goal of the autoresonant control system is to keep the displacement of the screen stable during the process of oscillation, while different loading conditions are induced. The influence of viscosity on control system performance is now considered. Figure 10(a) represents variations of viscosity over time, from $4 / 3 \mathrm{~N} . \mathrm{m} \mathrm{s}^{-1}$ to $8 / 3 \mathrm{~N}^{\mathrm{m} \mathrm{s}}{ }^{-1}$. The previous test $\left(10^{5} \mathrm{~s}\right)$ brought the system to a stable state after each viscosity increment. The response of the screen operation during the test is presented in Figure 10(b). The fluctuation response just after the viscosity changes shows that even small changes in the viscosity condition lead to changes in the performance of the system's vibration. Figure 10(c) shows the RMS value of the displacement signal (solid curve); the dashed line corresponds to the desired value of the RMS of the displacement signal. The desired value for this test is the same as for the previous test, $0.08 \mathrm{~m}$. When the RMS value leaves the phase control zone, the amplitude control starts. Figures 10(d) and (e) represent the phase control and amplitude control signal operation. When the amplitude control is applied and the amplitude value changed, the phase shift value remains unchanged. As soon as the RMS value enters the phase control zone, the amplitude control stops working and the phase control begins. 
(a)

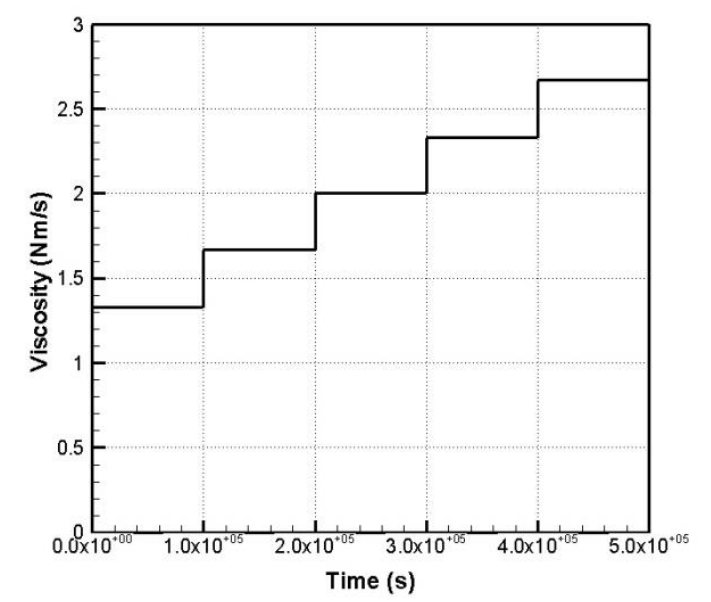

(b)
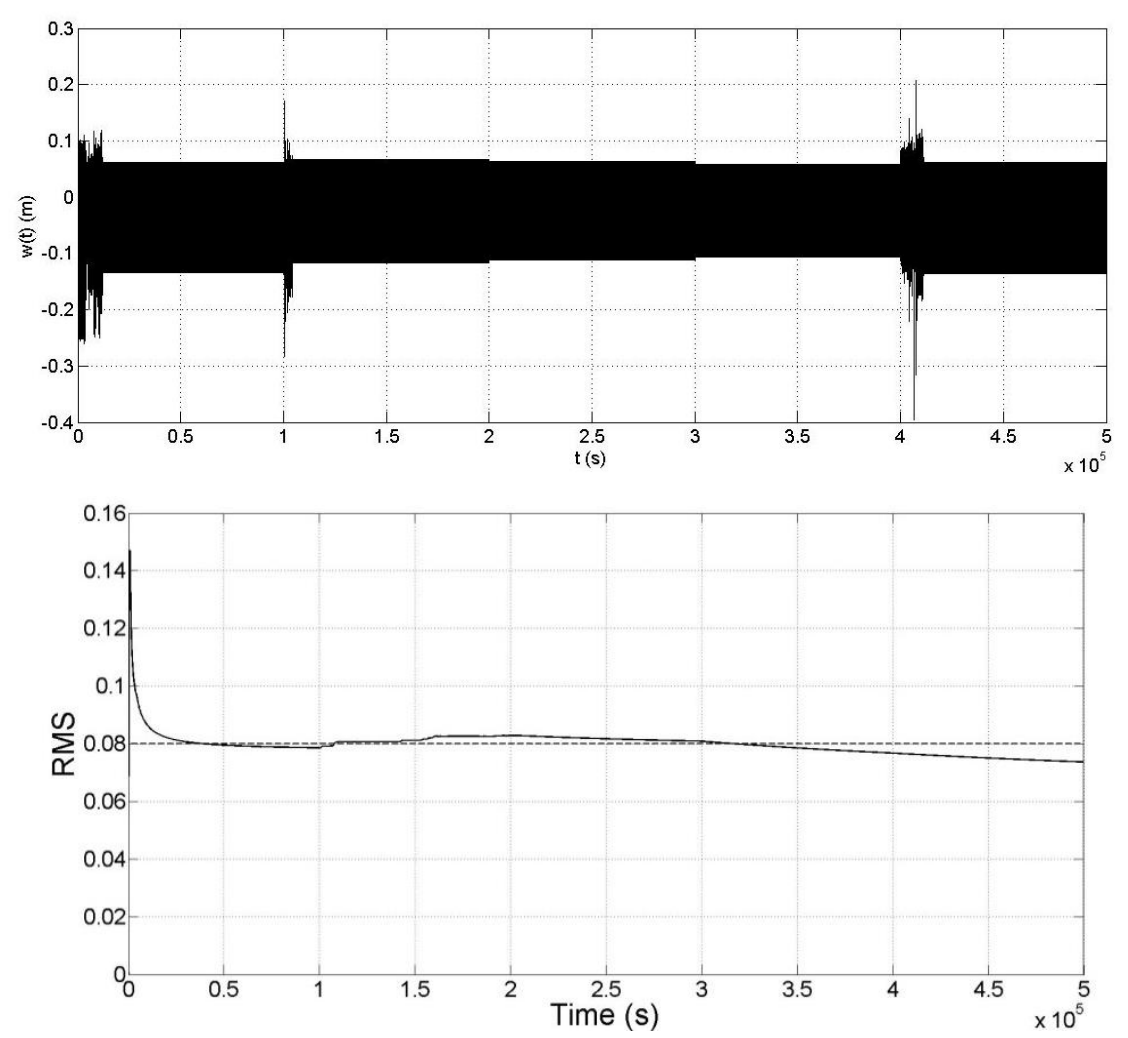

(d)

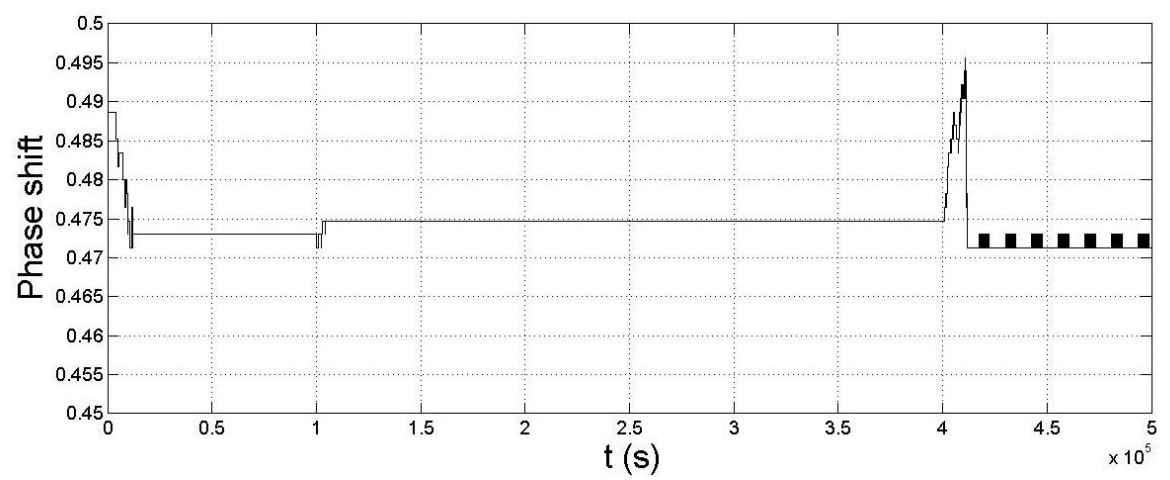


(e)

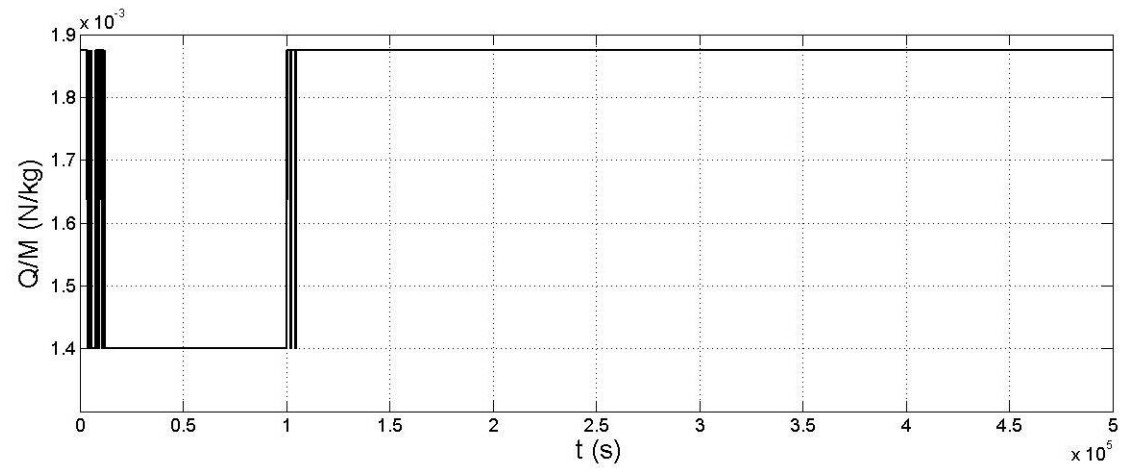

Figure 10: Performance of the control system, mass changing (a), displacement of the screen (b), RMS value (c), phase shift (d) and amplitude shift (e) of the displacement signal

This test shows the autoresonant control system keeping the displacement of the screen at the desired vibration level while as the viscosity varies between $4 / 3 \mathrm{~N} . \mathrm{m} \mathrm{s}^{-1}$ and $7 / 4 \mathrm{~N} . \mathrm{m} \mathrm{s}^{-1}$.

\section{Conclusions}

PR is a widespread phenomenon that may be threatening or beneficial according to the particular system where it takes place since very large oscillations can be generated by rather small parametric excitations. The exponentially growing response of the system creates awareness of the potential danger hidden in such a phenomenon. That is why a profound knowledge of the dynamics of systems where PR is to be induced is needed, in order to capture the energy flow between the different system modes when resonance takes place. Control strategies are designed to induce and stabilize unstable oscillations. In this paper autoresonant control was implemented for the intensification and stabilisation of a PR vibration screen in conditions of variable loading. The efficacy of the proposed control strategy has been verified in simulation, where both induction and stabilization of PR in screen machines have been successfully obtained. The results show that these characteristics retain robustness under a wide deviation of processing loads such as mass and viscosity uncertainties. Application of a robust and high-quality autoresonant control system offers the opportunity to increase productivity and efficiency, while improving design.

\section{Acknowledgement}

The work was performed with a financial support provided by FP7-People-2011-IAPP, Marie Curie Actions, Grant No. 281544. The model of the screen machine developed by L.Slepyan and 
V.Slepyan for the project was used as an object of control, V.Silberschmidt supported the investigation.

\section{References}

[1] V.K. Astashev, V.I. Babitsky, M.Z. Kolovsky, Dynamics and control of machines, Springer, Berlin, 2000.

[2] W .Ammann, H. Bachmann, Vibrations in structures: induced by man and machines, IABSEAIPC-IVBH, 1987.

[3] R.B. Randall, Vibration-based condition monitoring: industrial, aerospace and automotive applications, Wiley, New-York, 2011.

[4] V. Babitsky, Theory of vibro-impact systems and applications, Springer, Berlin, 1998.

[5] T.I. Fossen, H. Nijmeijer, Parametric resonance in dynamical systems, Springer Science \& Business Media, 2011.

[6] V. Slepyan, G. Loginov, L. Slepyan, The method of resonance excitation of a vibrating sieve and the vibrating screen for its implementation. Ukrainian Patent No. 87369, 2009.

[7] L. Slepyan, V. Slepyan, Coupled model parametric resonance in a vibrating screen, Mechanical Systems and Signal Processing 43 (2014) 295-304.

[8] W. Lacarbonara, H. Yabuno, K. Hayashi, Non-linear cancellation of the parametric resonance in elastic beams: Theory and experiment. International Journal of Solids and Structures 44 (7-8) (2007) 2209-2224.

[9] S.K. Dwivedy, R.C. Kar, Simultaneous combination, principal parametric and internal resonances in a slender beam with a lumped mass: three-mode interactions. Journal of Sound and Vibration 242 (1) (2001) 27-46.

[10] A.H. Nayfeh, Parametric excitation of two internally resonant oscillators. Journal of Sound and Vibration 119 (1987) 95-109.

[11] M. Okuma, H. Yabuno, N. Aoshima, Nonlinear bifurcation control of a parametrically excited cantilever beam. Proceedings of the 9th International Congress on Sound and Vibration, Paper No. 581 (2002).

[12] H. Yabuno, T. Murakami, J. Kawazoe, N. Aoshima, Suppression of parametric resonance in cantilever beam with a pendulum (effect of static friction at the supporting point of the pendulum). ASME Journal of Vibration and Acoustics 126 (2004) 149-162. 
[13] S. Oueini, A.H. Nayfeh, Single-mode control of a cantilever beam under principal parametric excitation. Journal of Sound and Vibration 224 (1999) 33-47.

[14] L. Chen, F. He, K. Sammut, Vibration suppression of a principal parametric resonance. Journal of Vibration and Control 15 (2009) 439-463.

[15] V. Babitsky, A.N. Kalashnikov, V.K. Astashev, Autoresonant control of nonlinear mode in ultrasonic transducer for machining applications. Ultrasonics 42 (2004) 29-35.

[16] V. Babitsky, A.N. Kalashnikov, F.V. Molodtsov, Autoresonant control of ultrasonically assisted cutting, Mechatronics 14(1) (2004) 91-114.

[17] V. Babitsky, J. Sokolov, Autoresonant homeostat concept for engineering application of nonlinear vibration modes, Nonlinear Dynamics 50 (3) (2007) 447-460.

[18] S. Voronina, V. Babitsky, Autoresonant control strategies of loaded ultrasonic transducer for machining applications. Journal of Sound and Vibration 313 (3-5) (2008) 395-417.

[19] V.K. Astashev, V.I. Babitsky, Ultrasonis Processes and Machines. Dynamics, Control and Applications, Springer, Berlin, 2007.

[20] X. Li, V. Babitsky, R.Parkin, A.Meadows, Autoresonant excitation and control of nonlinear mode for ultrasonically assisted drilling. ZAMM, 94 (2014) 904-910.

[21] F. Verhulst, Perturbation analysis of parametric resonance. Encyclopedia of Complexity and Systems Science. (2009) 6625-6639.

[22] S. Voronina, Modelling of autoresonant control of an ultrasonic transducer for machining applications, PhD thesis, 2008

[23] X. Li, Modelling and autoresonant control design of ultrasonically assisted drilling applications, $\mathrm{PhD}$ thesis, 2014 